Proyecciones Journal of Mathematics

Vol. 32, No 4, pp. 377-391, December 2013.

Universidad Católica del Norte

Antofagasta - Chile

\title{
Existence of positive periodic solutions for two types of second-order nonlinear neutral differential equations with variable delay
}

\author{
Abdelouaheb Ardjouni and Ahcene Djoudi \\ University of Annaba, Algeria \\ Received : January 2013. Accepted : September 2013
}

\begin{abstract}
In this article we study the existence of positive periodic solutions for two types of second-order nonlinear neutral differential equation with variable delay. The main tool employed here is the Krasnoselskii's fixed point theorem dealing with a sum of two mappings, one is a contraction and the other is completely continuous. The results obtained here generalize the work of Cheung, Ren and Han [7].

Subjclass : [2000] Primary 34K13, 34A34; Secondary 34K30, 34L30.

Keywords : Positive periodic solutions, nonlinear neutral differential equations, fixed point theorem.
\end{abstract}




\section{Introduction.}

Due to their importance in numerous applications, for example, physics, population dynamics, industrial robotics, and other areas, many authors are studying the existence, uniqueness, stability and positivity of solutions for delay differential equations, see the references in this article and references therein.

In this paper, we are interested in the analysis of qualitative theory of positive periodic solutions of delay differential equations. Motivated by the papers $[6,7,10,11,12,13,16,18,19]$ and the references therein, we concentrate on the existence of positive periodic solutions for the two types of second-order nonlinear neutral differential equation with variable delay

$$
\frac{d^{2}}{d t^{2}}(x(t)-g(t, x(t-\tau(t))))=a(t) x(t)-f(t, x(t-\tau(t)))
$$

and

$(1.2) \frac{d^{2}}{d t^{2}}(x(t)-g(t, x(t-\tau(t))))=-a(t) x(t)+f(t, x(t-\tau(t)))$,

where

$a, \tau \in C(\mathbf{R},(0, \infty)), g \in C(\mathbf{R} \times[0, \infty), \mathbf{R}), f \in C(\mathbf{R} \times[0, \infty),[0, \infty))$, and $a, \tau, g(t, x), f(t, x)$ are $T$-periodic in $t$ where $T$ is a positive constant. To reach our desired end we have to transform (1.1) and (1.2) into integral equations and then use Krasnoselskii's fixed point theorem to show the existence of positive periodic solutions. The obtained equation splits into a sum of two mappings, one is a contraction and the other is compact. In the special case $g(t, x)=c x$ with $|c|<1$, Cheung, Ren and Han in [7] show that (1.1) and (1.2) have a positive periodic solutions by using Krasnoselskii's fixed point theorem.

The organization of this paper is as follows. In Section 2, we introduce some notations and lemmas, and state some preliminary results needed in later sections, then we give the Green's function of (1.1) and (1.2), which plays an important role in this paper. Also, we present the inversions of (1.1) and (1.2), and Krasnoselskii's fixed point theorem. For details on Krasnoselskii's theorem we refer the reader to [17]. In Section 3 and Section 4 , we present our main results on existence of positive periodic solutions of (1.1) and (1.2), respectively. The results presented in this paper generalize the main results in [7]. 


\section{PRELIMINARIES}

For $T>0$, let $C_{T}$ be the set of all continuous scalar functions $x$, periodic in $t$ of period $T$. Then $\left(C_{T},\|\|.\right)$ is a Banach space with the supremum norm

$$
\|x\|=\sup _{t \in \mathbf{R}}|x(t)|=\sup _{t \in[0, T]}|x(t)| .
$$

Define

$$
C_{T}^{+}=\left\{x \in C_{T}: x>0\right\}, C_{T}^{-}=\left\{x \in C_{T}: x<0\right\} .
$$

Denote

$$
M=\sup \{a(t): t \in[0, T]\}, m=\inf \{a(t): t \in[0, T]\}, \beta=\sqrt{M},
$$

and

$$
F(t, x)=f(t, x(t-\tau(t)))-a(t) g(t, x(t-\tau(t))) .
$$

Lemma 2.1. ([7]) The equation

$$
\frac{d^{2}}{d t^{2}} y(t)-M y(t)=h(t), h \in C_{T}^{-},
$$

has a unique T-periodic solution

$$
y(t)=\int_{t}^{t+T} G_{1}(t, s)(-h(s)) d s
$$

where

$$
G_{1}(t, s)=\frac{\exp (-\beta(s-t))+\exp (\beta(s-t-T))}{2 \beta(1-\exp (-\beta T))}, s \in[t, t+T] .
$$

Lemma 2.2. ([7]) $G_{1}(t, s)>0$ and $\int_{t}^{t+T} G_{1}(t, s) d s=\frac{1}{M}$ for all $t \in[0, T]$ and $s \in[t, t+T]$.

Lemma 2.3. ([7]) The equation

$$
\frac{d^{2}}{d t^{2}} y(t)-a(t) y(t)=h(t), h \in C_{T}^{-},
$$

has a unique positive $T$-periodic solution

$$
\left(P_{1} h\right)(t)=\left(I-T_{1} B_{1}\right)^{-1} T_{1} h(t),
$$

where

$$
\left(T_{1} h\right)(t)=\int_{t}^{t+T} G_{1}(t, s)(-h(s)) d s,\left(B_{1} y\right)(t)=[-M+a(t)] y(t) .
$$


Lemma 2.4. ([7]) $P_{1}$ is completely continuous and satisfies

$$
0<\left(T_{1} h\right)(t) \leq\left(P_{1} h\right)(t) \leq \frac{M}{m}\left\|T_{1} h\right\|, h \in C_{T}^{-} .
$$

The following lemma is essential for our results on existence of positive periodic solution of (1.1).

Lemma 2.5. If $x \in C_{T}$ then $x$ is a solution of equation (1.1) if and only if

$x(t)=g(t, x(t-\tau(t)))+P_{1}(-f(t, x(t-\tau(t)))+a(t) g(t, x(t-\tau(t))))$.

Proof. Let $x \in P_{T}$ be a solution of (1.1). Rewrite (1.1) as

$$
\begin{gathered}
\frac{d^{2}}{d t^{2}}(x(t)-g(t, x(t-\tau(t))))-M(x(t)-g(t, x(t-\tau(t)))) \\
=(-M+a(t))(x(t)-g(t, x(t-\tau(t)))) \\
-f(t, x(t-\tau(t)))+a(t) g(t, x(t-\tau(t))) \\
=B_{1}(x(t)-g(t, x(t-\tau(t))))-f(t, x(t-\tau(t)))+a(t) g(t, x(t-\tau(t))) .
\end{gathered}
$$

From Lemma 2.1, we have

$$
\begin{aligned}
& x(t)-g(t, x(t-\tau(t)))=T_{1} B_{1}(x(t)-g(t, x(t-\tau(t)))) \\
& +T_{1}(-f(t, x(t-\tau(t)))+a(t) g(t, x(t-\tau(t)))) .
\end{aligned}
$$

This yields

$\left(I-T_{1} B_{1}\right)(x(t)-g(t, x(t-\tau(t))))$

$=T_{1}(-f(t, x(t-\tau(t)))+a(t) g(t, x(t-\tau(t))))$.

Therefore

$$
\begin{aligned}
& x(t)-g(t, x(t-\tau(t))) \\
& =\left(I-T_{1} B_{1}\right)^{-1} T_{1}(-f(t, x(t-\tau(t)))+a(t) g(t, x(t-\tau(t))))
\end{aligned}
$$


$=P_{1}(-f(t, x(t-\tau(t)))+a(t) g(t, x(t-\tau(t))))$.

Obviously

$x(t)=g(t, x(t-\tau(t)))+P_{1}(-f(t, x(t-\tau(t)))+a(t) g(t, x(t-\tau(t))))$.

This completes the proof.

Lemma 2.6. ([7]) The equation

$$
\frac{d^{2}}{d t^{2}} y(t)+M y(t)=h(t), h \in C_{T}^{+},
$$

has a unique T-periodic solution

$$
y(t)=\int_{t}^{t+T} G_{2}(t, s) h(s) d s,
$$

where

$$
G_{2}(t, s)=\frac{\cos \left(\beta\left(\frac{T}{2}+t-s\right)\right)}{2 \beta \sin \left(\frac{\beta T}{2}\right)}, s \in[t, t+T] .
$$

Lemma 2.7. ([7]) $\int_{t}^{t+T} G_{2}(t, s) d s=\frac{1}{M}$. Furthermore, if $M<\left(\frac{\pi}{T}\right)^{2}$, then $G_{2}(t, s)>0$ for all $t \in[0, T]$ and $s \in[t, t+T]$.

Lemma 2.8. ([7]) The equation

$$
\frac{d^{2}}{d t^{2}} y(t)+a(t) y(t)=h(t), h \in C_{T}^{+},
$$

has a unique positive $T$-periodic solution

$$
\left(P_{2} h\right)(t)=\left(I-T_{2} B_{2}\right)^{-1} T_{2} h(t),
$$

where

$$
\left(T_{2} h\right)(t)=\int_{t}^{t+T} G_{2}(t, s) h(s) d s,\left(B_{2} y\right)(t)=[M-a(t)] y(t) .
$$

Lemma 2.9. ([7]) $P_{2}$ is completely continuous. Furthermore, if $M<$ $\left(\frac{\pi}{T}\right)^{2}$, then

$$
0<\left(T_{2} h\right)(t) \leq\left(P_{2} h\right)(t) \leq \frac{M}{m}\left\|T_{2} h\right\|, h \in C_{T}^{+} .
$$


The following lemma is essential for our results on existence of positive periodic solution of (1.2).

Lemma 2.10. If $x \in C_{T}$ then $x$ is a solution of equation (1.2) if and only if

$x(t)=g(t, x(t-\tau(t)))+P_{2}(f(t, x(t-\tau(t)))-a(t) g(t, x(t-\tau(t))))$.

Proof. Let $x \in P_{T}$ be a solution of (1.2). Rewrite (1.2) as

$$
\begin{aligned}
& \frac{d^{2}}{d t^{2}}(x(t)-g(t, x(t-\tau(t))))+M(x(t)-g(t, x(t-\tau(t)))) \\
= & (M-a(t))(x(t)-g(t, x(t-\tau(t)))) \\
+ & f(t, x(t-\tau(t)))-a(t) g(t, x(t-\tau(t))) \\
= & B_{2}(x(t)-g(t, x(t-\tau(t))))+f(t, x(t-\tau(t)))-a(t) g(t, x(t-\tau(t))) .
\end{aligned}
$$

From Lemma 2.6, we have

$\mathrm{x}(t)-g(t, x(t-\tau(t)))=T_{2} B_{2}(x(t)-g(t, x(t-\tau(t))))$

$+T_{2}(f(t, x(t-\tau(t)))-a(t) g(t, x(t-\tau(t))))$.

This yields

$\left(I-T_{2} B_{2}\right)(x(t)-g(t, x(t-\tau(t))))$

$=T_{2}(f(t, x(t-\tau(t)))-a(t) g(t, x(t-\tau(t))))$.

Therefore

$\mathrm{x}(t)-g(t, x(t-\tau(t)))$

$=\left(I-T_{2} B_{2}\right)^{-1} T_{2}(f(t, x(t-\tau(t)))-a(t) g(t, x(t-\tau(t))))$

$=P_{2}(f(t, x(t-\tau(t)))-a(t) g(t, x(t-\tau(t))))$.

Obviously

$x(t)=g(t, x(t-\tau(t)))+P_{2}(f(t, x(t-\tau(t)))-a(t) g(t, x(t-\tau(t))))$. 
This completes the proof.

Lastly in this section, we state Krasnoselskii's fixed point theorem which enables us to prove the existence of positive periodic solutions to (1.1) and (1.2). For its proof we refer the reader to [17].

Theorem 2.11 (Krasnoselskii). Let $\mathbf{D}$ be a closed convex nonempty subset of a Banach space $(\mathbf{B},\|\|$.$) . Suppose that \mathcal{A}$ and $\mathcal{B}$ map $\mathbf{D}$ into B such that

(i) $x, y \in \mathbf{D}$, implies $\mathcal{A} x+\mathcal{B} y \in \mathbf{D}$,

(ii) $\mathcal{A}$ is completely continuous,

(iii) $\mathcal{B}$ is a contraction mapping.

Then there exists $z \in \mathbf{D}$ with $z=\mathcal{A} z+\mathcal{B} z$.

\section{POSITIVE PERIODIC SOLUTIONS FOR (1.1)}

To apply Theorem 2.11, we need to define a Banach space $\mathbf{B}$, a closed convex subset $\mathbf{D}$ of $\mathbf{B}$ and construct two mappings, one is a contraction and the other is a completely continuous. So, we let $(\mathbf{B},\|\cdot\|)=\left(C_{T},\|\cdot\|\right)$ and $\mathbf{D}=\{\varphi \in \mathbf{B}: L \leq \varphi \leq K\}$, where $L$ is non-negative constant and $K$ is positive constant. We express equation (2.1) as

$$
\varphi(t)=\left(\mathcal{B}_{1} \varphi\right)(t)+\left(\mathcal{A}_{1} \varphi\right)(t):=\left(H_{1} \varphi\right)(t),
$$

where $\mathcal{A}_{1}, \mathcal{B}_{1}: \mathbf{D} \rightarrow \mathbf{B}$ are defined by

$$
\left(\mathcal{A}_{1} \varphi\right)(t)=P_{1}(-f(t, \varphi(t-\tau(t)))+a(t) g(t, \varphi(t-\tau(t)))),
$$

and

$$
\left(\mathcal{B}_{1} \varphi\right)(t)=g(t, \varphi(t-\tau(t))) .
$$

In this section we obtain the existence of a positive periodic solution of (1.1) by considering the three cases;

$g(t, x)>0, g(t, x)=0$ and $g(t, x)<0$ for all $t \in \mathbf{R}, x \in \mathbf{D}$. We assume that function $g(t, x)$ is locally Lipschitz continuous in $x$. That is, there exists a positive constant $k$ such that

$$
|g(t, x)-g(t, y)| \leq k\|x-y\|, \text { for all } t \in[0, T], x, y \in \mathbf{D} .
$$

In the case $g(t, x)>0$, we assume that there exist positive constants $k_{1}$ and $k_{2}$ such that 


$$
k_{1} x \leq g(t, x) \leq k_{2} x, \text { for all } t \in[0, T], x \in \mathbf{D},
$$

and for all $t \in[0, T], x \in \mathbf{D}$,

$$
\left(1-k_{1}\right) k_{2} \leq F(t, x) \leq 1-k_{2} .
$$

Lemma 3.1. Suppose that (3.3) holds. If $\mathcal{B}_{1}$ is given by (3.2) with

$$
k<1,
$$

then $\mathcal{B}_{1}: \mathbf{D} \rightarrow \mathbf{B}$ is a contraction.

Proof. Let $\mathcal{B}_{1}$ be defined by (3.2). Obviously, $\mathcal{B}_{1} \varphi$ is continuous and it is easy to show that $\left(\mathcal{B}_{1} \varphi\right)(t+T)=\left(\mathcal{B}_{1} \varphi\right)(t)$. So, for any $\varphi, \psi \in \mathbf{D}$, we have

$$
\left|\left(\mathcal{B}_{1} \varphi\right)(t)-\left(\mathcal{B}_{1} \psi\right)(t)\right| \leq|g(t, \varphi(t-\tau(t)))-g(t, \psi(t-\tau(t)))|
$$
$\leq k\|\varphi-\psi\|$.

Then $\left\|\mathcal{B}_{1} \varphi-\mathcal{B}_{1} \psi\right\| \leq k\|\varphi-\psi\|$. Thus $\mathcal{B}_{1}: \mathbf{D} \rightarrow \mathbf{B}$ is a contraction by (3.7).

Besides, by the complete continuity of $P_{1}$, it is easy to verify the following lemma.

Lemma 3.2. Suppose that the conditions (3.4)-(3.6) hold. Then $\mathcal{A}_{1}$ : $\mathbf{D} \rightarrow \mathbf{B}$ is completely continuous.

Theorem 3.3. Suppose (3.3)-(3.7) hold with $L=\frac{k_{2}}{M}$ and $K=\frac{1}{m}$. Then equation (1.1) has a positive T-periodic solution $x$ in the subset

$$
\mathbf{D}=\left\{\varphi \in \mathbf{B}: \frac{k_{2}}{M} \leq \varphi \leq \frac{1}{m}\right\}
$$

Proof. By Lemma 3.1, the operator $\mathcal{B}_{1}: \mathbf{D} \rightarrow \mathbf{B}$ is a contraction. Also, from Lemma 3.2, the operator $\mathcal{A}_{1}: \mathbf{D} \rightarrow \mathbf{B}$ is completely continuous.

Moreover, we claim that $\mathcal{B}_{1} \psi+\mathcal{A}_{1} \varphi \in \mathbf{D}$ for all $\varphi, \psi \in \mathbf{D}$. Since $F(t, x) \geq\left(1-k_{1}\right) k_{2}>0$ which implies $-f(t, x)+a(t) g(t, x)<0$, then for any $\varphi, \psi \in \mathbf{D}$, by Lemma 2.2 and Lemma 2.4, we have 


$$
\begin{aligned}
& \left(\mathcal{B}_{1} \psi\right)(t)+\left(\mathcal{A}_{1} \varphi\right)(t) \\
& =g(t, \psi(t-\tau(t)))+P_{1}(-f(t, \varphi(t-\tau(t)))+a(t) g(t, \varphi(t-\tau(t)))) \\
& \leq k_{2} \psi(t-\tau(t))+\frac{M}{m}\left\|T_{1}(-f(t, \varphi(t-\tau(t)))+a(t) g(t, \varphi(t-\tau(t))))\right\| \\
& \leq \frac{k_{2}}{m}+\frac{M}{m} \max _{t \in[0, T]}\left|\int_{t}^{t+T} G_{1}(t, s)(f(s, \varphi(s-\tau(s)))-a(s) g(s, \varphi(s-\tau(s)))) d s\right| \\
& \leq \frac{k_{2}}{m}+\frac{M}{m} \max _{t \in[0, T]} \int_{t}^{t+T} G_{1}(t, s)(f(s, \varphi(s-\tau(s)))-a(s) g(s, \varphi(s-\tau(s)))) d s \\
& \leq \frac{k_{2}}{m}+\frac{M}{m} \int_{t}^{t+T} G_{1}(t, s)\left(1-k_{2}\right) d s \\
& \leq \frac{k_{2}}{m}+\frac{M}{m}\left(1-k_{2}\right) \frac{1}{M} \\
& =\frac{1}{m} .
\end{aligned}
$$

On the other hand, Lemma 2.2 and Lemma 2.4,$$
\left(\mathcal{B}_{1} \psi\right)(t)+\left(\mathcal{A}_{1} \varphi\right)(t)
$$$$
=g(t, \psi(t-\tau(t)))+P_{1}(-f(t, \varphi(t-\tau(t)))+a(t) g(t, \varphi(t-\tau(t))))
$$$$
\geq k_{1} \psi(t-\tau(t))+\int_{t}^{t+T} G_{1}(t, s)(f(s, \varphi(s-\tau(s)))-a(s) g(s, \varphi(s-\tau(s)))) d s
$$$$
\geq \frac{k_{1} k_{2}}{M}+\int_{t}^{t+T} G_{1}(t, s)\left(1-k_{1}\right) k_{2} d s
$$$$
=\frac{k_{1} k_{2}}{M}+\left(1-k_{1}\right) k_{2} \frac{1}{M}
$$$$
=\frac{k_{2}}{M} \text {. }
$$

Then $\mathcal{B}_{1} \psi+\mathcal{A}_{1} \varphi \in \mathbf{D}$ for all $\varphi, \psi \in \mathbf{D}$. Clearly, all the hypotheses of the Krasnoselskii theorem are satisfied. Thus there exists a fixed point $x \in \mathbf{D}$ such that $x=\mathcal{A}_{1} x+\mathcal{B}_{1} x$. By Lemma 2.5 this fixed point is a solution of (1.1) and the proof is complete.

Remark 3.4. When $g(t, x)=c x$, Theorem 3.3 reduces to Theorem 2.1 of [7].

In the case $g(t, x)=0$, we have 5 theorem. 
Theorem 3.5. ([7]) If $k_{2}=0$ and $0<F(t, x) \leq 1$, then equation (1.1) has a positive T-periodic solution $x$ in the subset $\mathbf{D}_{1}=\left\{\varphi \in \mathbf{B}: 0<\varphi \leq \frac{1}{m}\right\}$.

In the case $g(t, x)<0$, we substitute conditions (3.4)-(3.6) with the following conditions respectively. We assume that there exist negative constants $k_{3}$ and $k_{4}$ such that

$$
\begin{gathered}
k_{3} x \leq g(t, x) \leq k_{4} x, \text { for all } t \in[0, T], x \in \mathbf{D}, \\
-k_{3}<\frac{m}{M}
\end{gathered}
$$

and for all $t \in[0, T], x \in \mathbf{D}$

$$
-k_{3} M<F(t, x) \leq m .
$$

Theorem 3.6. Suppose (3.3) and (3.7)-(3.10) hold with $L=0$ and $K=$ 1. Then equation (1.1) has a positive T-periodic solution $x$ in the subset $\mathbf{D}_{2}=\{\varphi \in \mathbf{B}: 0<\varphi \leq 1\}$.

Proof. By Lemma 3.1, the operator $\mathcal{B}_{1}: \mathbf{D} \rightarrow \mathbf{B}$ is a contraction. Also, from Lemma 3.2, the operator $\mathcal{A}_{1}: \mathbf{D} \rightarrow \mathbf{B}$ is completely continuous. Moreover, we claim that $\mathcal{B}_{1} \psi+\mathcal{A}_{1} \varphi \in \mathbf{D}$ for all $\varphi, \psi \in \mathbf{D}$. In fact, for any $\varphi, \psi \in \mathbf{D}$, by Lemma 2.2 and Lemma 2.4, we have

$$
\begin{aligned}
& \left(\mathcal{B}_{1} \psi\right)(t)+\left(\mathcal{A}_{1} \varphi\right)(t) \\
& =\mathrm{g}(t, \psi(t-\tau(t)))+P_{1}(-f(t, \varphi(t-\tau(t)))+a(t) g(t, \varphi(t-\tau(t)))) \\
& \leq k_{4} \psi(t-\tau(t))+\frac{M}{m}\left\|T_{1}(-f(t, \varphi(t-\tau(t)))+a(t) g(t, \varphi(t-\tau(t))))\right\| \\
& \leq \frac{M}{m} \max _{t \in[0, T]}\left|\int_{t}^{t+T} G_{1}(t, s)(f(s, \varphi(s-\tau(s)))-a(s) g(s, \varphi(s-\tau(s)))) d s\right| \\
& \leq \frac{M}{m} \max _{t \in[0, T]} \int_{t}^{t+T} G_{1}(t, s)(f(s, \varphi(s-\tau(s)))-a(s) g(s, \varphi(s-\tau(s)))) d s \\
& =\frac{M}{m} \int_{t}^{t+T} G_{1}(t, s) m d s \\
& =\frac{M}{m} m \frac{1}{M}
\end{aligned}
$$

$=1$.

On the other hand, Lemma 2.2 and Lemma 2.4, 


$$
\begin{aligned}
& \left(\mathcal{B}_{1} \psi\right)(t)+\left(\mathcal{A}_{1} \varphi\right)(t) \\
& =\mathrm{g}(t, \psi(t-\tau(t)))+P_{1}(-f(t, \varphi(t-\tau(t)))+a(t) g(t, \varphi(t-\tau(t)))) \\
& \geq k_{3} \psi(t-\tau(t))+\int_{t}^{t+T} G_{1}(t, s)(f(s, \varphi(s-\tau(s)))-a(s) g(s, \varphi(s-\tau(s)))) d s \\
& \geq k_{3}+\int_{t}^{t+T} G_{1}(t, s)\left(-k_{3} M\right) d s \\
& =k_{3}+\left(-k_{3} M\right) \frac{1}{M} \\
& =0 .
\end{aligned}
$$

Then $\mathcal{B}_{1} \psi+\mathcal{A}_{1} \varphi \in \mathbf{D}$ for all $\varphi, \psi \in \mathbf{D}$. Clearly, all the hypotheses of the Krasnoselskii theorem are satisfied. Thus there exists a fixed point $x \in \mathbf{D}$ such that $x=\mathcal{A}_{1} x+\mathcal{B}_{1} x$. Since $F(t, x)>-k_{3} M$, it is clear that $x(t)>0$, hence $x \in \mathbf{D}_{2}$. By Lemma 2.5 this fixed point is a solution of (1.1) and the proof is complete.

Remark 3.7. When $g(t, x)=c x$, Theorem 3.6 reduces to Theorem 2.3 of [7].

\section{POSITIVE PERIODIC SOLUTIONS FOR (1.2)}

We express equation (2.2) as

$$
\varphi(t)=\left(\mathcal{B}_{2} \varphi\right)(t)+\left(\mathcal{A}_{2} \varphi\right)(t):=\left(H_{2} \varphi\right)(t),
$$

where $\mathcal{A}_{2}, \mathcal{B}_{2}: \mathbf{D} \rightarrow \mathbf{B}$ are defined by

$$
\left(\mathcal{A}_{2} \varphi\right)(t)=P_{2}(f(t, \varphi(t-\tau(t)))-a(t) g(t, \varphi(t-\tau(t))))
$$

and

$$
\left(\mathcal{B}_{2} \varphi\right)(t)=g(t, \varphi(t-\tau(t))) .
$$

Moreover, by the complete continuity of $P_{2}$, it is easy to verify

Lemma 4.1. Suppose that the conditions (3.4)-(3.6) hold. Then $\mathcal{A}_{2}$ : $\mathbf{D} \rightarrow \mathbf{B}$ is completely continuous.

Remark 4.2. Notice that $\mathcal{B}_{2}$ in this section is defined exactly the same as that in Section 3. Hence Lemma 3.1 still holds true. 
Similar to the results in Section 3, we have

Theorem 4.3. Assume that the hypotheses of Theorem 3.3 hold. Also assume that

$$
\max \{a(t): t \in[0, T]\}<\left(\frac{\pi}{T}\right)^{2} .
$$

Then equation (1.2) has a positive T-periodic solution $x$ in the subset $\mathbf{D}=\left\{\varphi \in \mathbf{B}: \frac{k_{2}}{M} \leq \varphi \leq \frac{1}{m}\right\}$.

Theorem 4.4. ([7]) Assume that the hypotheses of Theorem 3.5 hold. Also assume that

$$
\max \{a(t): t \in[0, T]\}<\left(\frac{\pi}{T}\right)^{2}
$$

Then equation (1.2) has a positive T-periodic solution $x$ in the subset $\mathbf{D}_{1}=\left\{\varphi \in \mathbf{B}: 0<\varphi \leq \frac{1}{m}\right\}$.

Theorem 4.5. Assume that the hypotheses of Theorem 3.6 hold. Also assume that

$$
\max \{a(t): t \in[0, T]\}<\left(\frac{\pi}{T}\right)^{2} .
$$

Then equation (1.2) has a positive T-periodic solution $x$ in the subset $\mathbf{D}_{2}=\{\varphi \in \mathbf{B}: 0<\varphi \leq 1\}$.

Remark 4.6. When $g(t, x)=c x$, Theorem 4.3 and Theorem 4.5 reduce to Theorem 3.1 and Theorem 3.3 of [7], respectively.

Acknowledgement. The authors are grateful to the referee for his/ her comments and good advice.

\section{References}

[1] A. Ardjouni and A. Djoudi, Existence of periodic solutions for nonlinear neutral dynamic equations with variable delay on a time scale. Commun Nonlinear Sci Numer Simulat 17, pp. 3061-3069, (2012). 
[2] A. Ardjouni and A. Djoudi, Periodic solutions for a second-order nonlinear neutral differential equation with variable delay, Electronic Journal of Differential Equations, Vol. No. 128, pp. 1-7, (2011).

[3] A. Ardjouni and A. Djoudi, Periodic solutions in totally nonlinear dynamic equations with functional delay on a time scale, Rend. Sem. Mat. Univ. Politec. Torino Vol. 68, 4, pp. 349-359, (2010).

[4] T. A. Burton, Liapunov functionals, fixed points and stability by Krasnoselskii's theorem. Nonlinear Stud. 9(2002), No. 2, 181-190.

[5] T. A. Burton, Stability by Fixed Point Theory for Functional Differential Equations, Dover Publications, New York, 2006.

[6] F. D. Chen, Positive periodic solutions of neutral Lotka-Volterra system with feedback control, Appl. Math. Comput. 162, no. 3, pp. 1279$1302,(2005)$.

[7] W. S. Cheung, J. Ren and W. Han, Positive periodic solutions of second order neutral functional differential equations, Nonlinear Analysis 71, pp. 3948-3955, (2009).

[8] H. Deham and A. Djoudi, Periodic solutions for nonlinear differential equation with functional delay, Georgian Mathematical Journal 15, No. 4, pp. 635-642, (2008).

[9] H. Deham and A. Djoudi, Existence of periodic solutions for neutral nonlinear differential equations with variable delay, Electronic Journal of Differential Equations, Vol. No. 127, pp. 1-8, (2010).

[10] Y. M. Dib, M. R. Maroun and Y. N. Raffoul, Periodicity and stability in neutral nonlinear differential equations with functional delay, Electronic Journal of Differential Equations, Vol. No. 142, pp. 1-11, (2005).

[11] E. R. Kaufmann, A nonlinear neutral periodic differential equation, Electron. J. Differential Equations, No. 88, pp. 1-8, (2010).

[12] Y. Liu and W. Ge, Positive periodic solutions of nonlinear duffing equations with delay and variable coefficients, Tamsui Oxf. J. Math. Sci. 20, pp. 235-255, (2004). 
[13] Y. Luo, W. Wang and J. Shen, Existence of positive periodic solutions for two kinds of neutral functional differential equations, Applied Mathematics Letters 21, pp. 581-587, (2008).

[14] Y. N. Raffoul, Periodic solutions for neutral nonlinear differential equations with functional delay, Electron. J. Differential Equations, No. 102, pp. 1-7, (2003).

[15] Y. N. Raffoul, Stability in neutral nonlinear differential equations with functional delays using fixed-point theory, Math. Comput. Modelling 40, No. 7-8, pp. 691-700, (2004).

[16] Y. N. Raffoul, Positive periodic solutions in neutral nonlinear differential equations, E. J. Qualitative Theory of Diff. Equ., No. 16, pp. 1-10, (2007).

[17] D. S. Smart, Fixed point theorems; Cambridge Tracts in Mathematics, No. 66. Cambridge University Press, London-New York, (1974).

[18] Q. Wang, Positive periodic solutions of neutral delay equations (in Chinese), Acta Math. Sinica (N.S.) 6, pp. 789-795, (1996).

[19] E. Yankson, Positive periodic solutions for second-order neutral differential equations with functional delay, Electron. J. Differential Equations, No. 14, pp. 1-6, (2012).

[20] W. Zeng, Almost periodic solutions for nonlinear Duffing equations, Acta Math. Sinica (N. S.) 13, pp. 373-380, (1997).

\section{A. Ardjouni}

Department of Mathematics, Faculty of Sciences

University of Annaba, P. O. Box 12 Annaba, Algeria

e-mail : abd_ardjouni@yahoo.fr

and 


\section{A. Djoudi}

Department of Mathematics,

Faculty of Sciences

University of Annaba,

P. O. Box 12 Annaba,

Algeria

e-mail : adjoudi@yahoo.com 